\title{
Equitable hypergraph orientations
}

\section{Yair Caro * $\quad$ Douglas West ${ }^{\dagger} \quad$ Raphael Yuster ${ }^{\ddagger}$}

Submitted: Dec 14, 2010; Accepted: May 13, 2011; Published: May 23, 2011

Mathematics Subject Classification: 05C65

\begin{abstract}
A classical result in graph theory asserts that every graph can be oriented so that the indegree and outdegree of each vertex differ by at most 1 . We study the extent to which the result generalizes to uniform hypergraphs.
\end{abstract}

\section{Introduction}

In recent years, many researchers have begun to study hypergraph generalizations of classical results in graph theory. Areas of study include Turán-type problems, matchings and factors, and properties of random structures. In this spirit, we consider the generalization of an elementary classical result.

An orientation of a graph is obtained by assigning a direction (order) to each edge. An orientation is fully balanced if each vertex has the same number of edges entering it and exiting it. An even graph is a graph whose vertex degrees are all even. The existence of Eulerian circuits in connected even graphs implies that all even graphs have fully balanced orientations. An easy consequence is that every graph has an equitable orientation, where an orientation is equitable if for every vertex the numbers of entering and exiting edges differ by at most 1 .

In this note we generalize equitable orientation to the setting of uniform hypergraphs. An orientation of a hypergraph associates with each edge an ordering of its vertices; an edge of size $r$ can be ordered in $r$ ! ways, with each vertex in one of $r$ positions. Let $\vec{H}$ denote an orientation of an $r$-uniform hypergraph $H$. For a set $S$ of positions and a set

\footnotetext{
*Department of Mathematics, University of Haifa-Oranim, Tivon 36006, Israel: yacaro@kvgeva.org.il.

${ }^{\dagger}$ Department of Mathematics, University of Illinois, Urbana, IL, 61801, U.S.A.: west@math.uiuc.edu. Research supported by the National Security Agency under Award H98230-10-1-0363.

${ }^{\ddagger}$ Department of Mathematics, University of Haifa, Haifa 31905, Israel: raphy@math.haifa.ac.il.
} 
$U$ of vertices in $H$, with $|U|=|S|$, let $d_{S}(U)$ denote the number of edges in $\vec{H}$ in which the set of positions occupied by $U$ is precisely $S$.

For $p \in\{1, \ldots, r\}$, say that an orientation $\vec{H}$ of an $r$-uniform hypergraph $H$ is $p$ equitable if $\left|d_{S}(U)-d_{T}(U)\right| \leq 1$ for every $p$-set $U$ of vertices and $p$-sets $S$ and $T$ of positions. The statement that every graph has an equitable orientation is just the statement that every 2-uniform hypergraph has a 1-equitable orientation. We present three results about p-equitable orientations of hypergraphs.

Theorem 1. For $p \in\{1, r-1\}$, every $r$-uniform hypergraph has a $p$-equitable orientation.

For 3-uniform hypergraphs, Theorem 1 covers all cases. For larger $r$, Theorem 1 is essentially the best one can prove. Already for $r=4$, there are 4-uniform hypergraphs having no 2-equitable orientation. Our second theorem yields a more general family of examples. Let $K_{n}^{r}$ denote the complete $r$-uniform hypergraph with $n$ vertices.

Theorem 2. If $1<p<r-1$ and $\left(\begin{array}{l}r \\ p\end{array}\right)$ divides $\left(\begin{array}{l}n-p \\ r-p\end{array}\right)$, then existence of a p-equitable orientation of $K_{n}^{r}$ requires that $r$ divides $\left(\begin{array}{l}n-1 \\ r-1\end{array}\right)$.

As a corollary, we show that if $p$ is a prime power and $m$ is a positive integer relatively prime to $p$, then $K_{(m+1) p}^{m p}$ has no $p$-equitable orientation. Also, $K_{n}^{4}$ has no 2-equitable orientation when $n \equiv 6 \bmod 12$.

Although $p$-equitable orientations may fail to exist when $1<p<r-1$, they do exist when each edge shares fewer than $p$ elements with most other edges. The $p$-set multigraph $L_{p}(H)$ of a hypergraph $H$ is the graph whose vertices are the edges of $H$, such that edges $e$ and $e^{\prime}$ in $H$ are adjacent in $L_{p}(H)$ if they have at least $p$ common vertices. The multiplicity of the resulting edge in $L_{p}(H)$ is the number of $p$-subsets of $e \cap e^{\prime}$. When $H$ is 2-uniform, $L_{1}(H)$ is simply the line graph of $H$. We prove that if the maximum degree of $L_{p}(H)$ is relatively small, then $H$ has a $p$-equitable orientation. Indeed, it suffices to bound the degeneracy; a graph is $k$-degenerate if every subgraph has a vertex of degree at most $k$.

Theorem 3. Let $H$ be an r-uniform hypergraph. If $L_{p}(H)$ is $\left(\left(\begin{array}{l}r \\ p\end{array}\right)-1\right)$-degenerate, then $H$ has a p-equitable orientation. In particular, if $\Delta\left(L_{p}(H)\right)<\left(\begin{array}{l}r \\ p\end{array}\right)$, then $H$ has a p-equitable orientation.

Section 2 contains the proofs; Section 3 presents some extensions and open problems. There are several ways to prove Theorem 1, extending various proofs of the special case $r=2$. We present a proof based on König's Theorem for edge-colorings of bipartite graphs (see [2, p. 37], for example): every bipartite graph with maximum degree $r$ decomposes into $r$ matchings. 


\section{Proofs}

We write $V(H)$ and $E(H)$ for the vertex set and edge set of a hypergraph $H$. Let $[r]=\{1, \ldots, r\}$. If $|U| \leq r$, then $\sum d_{S}(U)=d(U)$, where the sum is over all subsets $S$ of $[r]$ with $|S|=|U|$, and $d(U)$ denotes the number of edges in $H$ containing $U$.

Theorem (1). For $p \in\{1, r-1\}$, every $r$-uniform hypergraph has a p-equitable orientation.

Proof. Let $H$ be an $r$-uniform hypergraph. Let $X$ be the family of $p$-sets in $V(H)$, and let $Y=E(H)$. Let $B$ be the bipartite graph with partite sets $X$ and $Y$ such that vertices $U \in X$ and $e \in Y$ are adjacent in $B$ if and only if $U \subseteq e$. Since $p \in\{1, r-1\}$, the degree in $B$ of each element of $Y$ is $r$, and the degree in $B$ of an element $U$ of $X$ is its degree in $H$, which we have written as $d(U)$.

Form a modified graph $B^{\prime}$ from $B$ as follows. Split each $U \in X$ into $\lceil d(U) / r\rceil$ vertices and assign each edge incident to $U$ to one of these copies of $U$ so that each copy except possibly the last is incident to $r$ edges in $B^{\prime}$. Thus $B^{\prime}$ is a bipartite graph with maximum degree $r$. By König's Theorem, $B^{\prime}$ decomposes into $r$ matchings $M_{1}, \ldots, M_{r}$.

From $M_{1}, \ldots, M_{r}$, we define an orientation $\vec{H}$. The neighbors in $B^{\prime}$ of a vertex $e \in Y$ are copies of distinct vertices of $X$, since $B$ has no multi-edges. The edges of $B^{\prime}$ incident to $e$ lie in distinct matchings in $B^{\prime}$. For $U e \in M_{i}$, in position $i$ of the ordering of $e$ in $\vec{H}$ put

a) the one element of $U$ if $p=1$.

b) the one element of $e-U$ if $p=r-1$.

In the latter case, $U$ occupies the set $[r]-\{i\}$ of positions in the ordering of $e$.

Since each copy of $U$ is incident to at most one edge in each $M_{i}$, and there are $\lceil d(U) / r\rceil$ copies of $U$, each $U$ appears in each of the $r$ possible (sets of) positions at most $\lceil d(U) / r\rceil$ times. Furthermore, since each copy of $U$ except the last has degree $r$ in $B^{\prime}$, each possibility occurs at least $\lceil d(U) / r\rceil-1$ times. Hence $\vec{H}$ is $p$-equitable.

Theorem (2). If $1<p<r-1$, and $\left(\begin{array}{l}r \\ p\end{array}\right)$ divides $\left(\begin{array}{l}n-p \\ r-p\end{array}\right)$, then a necessary condition for $K_{n}^{r}$ having a p-equitable orientation is that $r$ divides $\left(\begin{array}{l}n-1 \\ r-1\end{array}\right)$.

Proof. Consider a $p$-equitable orientation of $K_{n}^{r}$. Write it as a matrix, with the vertices of each ordered edge as a row in a matrix with $\left(\begin{array}{l}n \\ r\end{array}\right)$ rows and $r$ columns. Each $p$-set of elements appears in $\left(\begin{array}{l}n-p \\ r-p\end{array}\right)$ rows. By the divisibility and equitability hypotheses, each $p$-set of elements appears exactly $\left(\begin{array}{l}n-p \\ r-p\end{array}\right) /\left(\begin{array}{l}r \\ p\end{array}\right)$ times in each $p$-set of columns. Let $s$ be this ratio.

Since each $p$-set of vertices occurs $s$ times in a fixed $p$-set of columns, a given vertex $z$ appears $s\left(\begin{array}{l}n-1 \\ p-1\end{array}\right)$ times in these columns. Thus $z$ appears the same number of times in each 
$p$-set of columns; call this number $t$. Since $z$ appears $t$ times in its $p$ most frequent columns and also $t$ times in its $p$ least frequent columns, $z$ must appear the same number of times in each of the $r$ columns. Since $z$ appears in exactly $\left(\begin{array}{l}n-1 \\ r-1\end{array}\right)$ rows, $r$ divides $\left(\begin{array}{l}n-1 \\ r-1\end{array}\right)$.

Corollary 4. For $m, s \in \mathbb{N}$ and $q$ prime, let $p=q^{s}$. If $q \nmid m$, then $K_{(m+1) p}^{m p}$ has no p-equitable orientation. Also, $K_{n}^{4}$ has no 2 -equitable orientation when $n \equiv 6 \bmod 12$.

Proof. Let $n=(m+1) p$ and $r=m p$. Since $\left(\begin{array}{l}n-p \\ r-p\end{array}\right)=\left(\begin{array}{c}r \\ r-p\end{array}\right)=\left(\begin{array}{l}r \\ p\end{array}\right)$, the divisibility hypothesis of Theorem 2 holds. Also, $\left(\begin{array}{c}n-1 \\ r-1\end{array}\right)=\left(\begin{array}{c}n-1 \\ n-r\end{array}\right)=\left(\begin{array}{c}n-1 \\ p\end{array}\right)$. Since $q$ does not divide $m$, the product $\prod_{i=1}^{p}(n-i)$ has no factor divisible by $q^{s+1}$. Hence it has $q^{s-i}$ factors divisible by $q^{i}$, for $1 \leq i \leq s$, and the exponent on $q$ in the prime factorization of $\prod_{i=1}^{p}(n-i)$ is $\left(q^{s}-1\right) /(q-1)$. The exponent on $q$ is the same in the prime factorization of $p$ !. Hence $q$ does not divide $\left(\begin{array}{c}n-1 \\ p\end{array}\right)$, so also $m p$ does not divide $\left(\begin{array}{c}n-1 \\ p\end{array}\right)$. The necessary condition of Theorem 2 fails.

If $r=4$ and $p=2$, then $\left(\begin{array}{l}n-p \\ r-p\end{array}\right)=(n-2)(n-3) / 2$ and $\left(\begin{array}{l}r \\ p\end{array}\right)=6$. The divisibility hypothesis holds when $n$ is congruent to 6 or 11 modulo 12 . The necessary condition for $p$-equitable orientation is that 4 divides $\left(\begin{array}{c}n-1 \\ 3\end{array}\right)$; this fails when $n \equiv 6 \bmod 12$.

The proof of Theorem 2 holds also when $p \in\{1, r-1\}$, but in these cases the hypothesis implies the divisibility requirement. When $p=1$, the conditions are the same. When $p=r-1$, the hypothesis is that $r$ divides $n-r+1$. Let $k=(n-r+1) / r$. In this case, $r$ divides $\left(\begin{array}{c}k r+r-2 \\ r-1\end{array}\right)$; study the primes dividing $r$ in the numerator and denominator, noting that the smallest factor in the numerator is divisible by $r$.

It is interesting to note that when $r$ divides $n-r+1$, every $(r-1)$-equitable orientation of $K_{n}^{r}$ is also 1-equitable. Surprisingly, the converse is not true. The first orientation of $K_{5}^{3}$ below is 1-equitable but not 2-equitable; the second is 2-equitable.

$$
\begin{aligned}
& \{(123),(124),(512),(431),(315),(451),(234),(253),(542),(345)\} \\
& \{(123),(142),(512),(314),(531),(451),(234),(253),(425),(345)\}
\end{aligned}
$$

Finally, we prove a sufficient condition in terms of sparseness.

Theorem (3). Let $H$ be an r-uniform hypergraph. If $L_{p}(H)$ is $\left(\left(\begin{array}{l}r \\ p\end{array}\right)-1\right)$-degenerate, then $H$ has a p-equitable orientation. (This holds in particular when $\Delta\left(L_{p}(H)\right)<\left(\begin{array}{l}r \\ p\end{array}\right)$.)

Proof. By Theorem 1, we may assume $1<p<r-1$. Since every subgraph of $L_{p}(H)$ has a vertex with degree less than $\left(\begin{array}{l}r \\ p\end{array}\right)$, a "least-degree-last" ordering indexes the vertices of $L_{p}(H)$ (edges of $H$ ) as $\left\{e_{1}, \ldots, e_{t}\right\}$ so that for each $i$, fewer than $\left(\begin{array}{l}r \\ p\end{array}\right)$ pairs $(P, j)$ satisfy $P \subseteq e_{i} \cap e_{j}$ with $|P|=p$ and $j<i$. Let $R_{i}$ denote this set of pairs. 
We iteratively orient the edges of $H$ from $e_{1}$ through $e_{t}$ so that no $p$-set occupies the same set of positions more than once; such an orientation is equitable. Orient $e_{1}$ arbitrarily. Having oriented $e_{1}, \ldots, e_{i-1}$ with the desired property, each pair $(P, j) \in R_{i}$ forbids $p !(r-p)$ ! orderings for $e_{i}$. Since $\left|R_{i}\right|<\left(\begin{array}{l}r \\ p\end{array}\right)$, the total number of forbidden orderings is less than $r$ !, and a good ordering for $e_{i}$ exists.

Instead of storing all excluded orderings to find a good ordering for $e_{i}$, we can compute the desired ordering position by position. Among the candidates to occupy position 1 in $e_{i}$, there is one such that the number of orderings of the rest of $e_{i}$ forbidden by earlier edges is less than $(r-1)$ !. This iterates through the positions to yield an efficient algorithm.

Both the proof of Theorem 3 and this algorithm can be viewed probabilistically. The probability that a random ordering of $e_{i}$ is forbidden by the earlier edges is less than 1. Converting this to a deterministic algorithm via the computation described above is called the method of conditional expectations (cf. [1]): position by position, we choose the next element of the ordering so that the probability that set of positions at contains a forbidden $p$-set under random completion of the ordering remains below 1 .

\section{$3 \quad$ Extensions and open problems}

It seems plausible that an $r$-uniform hypergraph $H$ should have a $p$-equitable orientation when $r$ is not too small compared to $p$ and to the maximum degree among $p$-sets. Formally, let $\Delta_{p}(H)=\max \{d(U):|U|=p\}$.

Conjecture 5. Given positive integers $p$ and $k$, there exists $r_{0}$ such that if $r>r_{0}$, then every $r$-uniform hypergraph $H$ with $\Delta_{p}(H) \leq k$ has a p-equitable orientation.

The case $p=1$ of Conjecture 5 holds by Theorem 1, the case $k=1$ trivially holds, and the case $k=2$ "almost" holds since then $\Delta\left(L_{p}(H)\right) \leq\left(\begin{array}{l}r \\ p\end{array}\right)$.

We prove a relaxed version of Conjecture 5. Say that an orientation of an $r$-uniform hypergraph $H$ is nearly p-equitable if $\left|d_{S}(U)-d_{T}(U)\right| \leq 2$ whenever $U \subset V(H)$ and $S, T \subset[r]$ with $|U|=|S|=|T|=p$. That is, the $p$-set occupancies may vary by up to 2 , as opposed to 1 in a $p$-equitable orientation. Similarly to Theorem 3 , a sparseness condition guarantees an ordering in which every $p$-set of vertices occupies each $p$-set of positions at most twice.

Theorem 6. For positive integers $p$, $k$, and $r$ such that $\left(\begin{array}{c}r \\ p\end{array}\right)>9\left(\begin{array}{c}k-1 \\ 2\end{array}\right)$, every $r$-uniform hypergraph $H$ with $\Delta_{p}(H) \leq k$ has a nearly p-equitable orientation. 
Proof. Orient $H$ randomly by choosing the ordering of each edge uniformly and independently. For $U \subset V(H)$ with $|U|=p$ and any set of three edges $\{e, f, g\}$ that all contain $U$, let $A(U,\{e, f, g\})$ denote the event that $U$ occupies the same $p$ positions in all three edges. It suffices to prove that with positive probability, no event of this form holds.

The probability of $A(U,\{e, f, g\})$ is the probability that in both $f$ and $g$ the positions occupied by $U$ are the same as the positions they occupy in $e$. Since the edge-orderings are independent, this probability is $\left(\begin{array}{l}r \\ p\end{array}\right)^{-2}$.

Let $F$ be the family of events specified by triples avoiding $\{e, f, g\}$. Since edgeorderings are chosen independently, $A(U,\{e, f, g\})$ is mutually independent of $F$. To bound the number of events excluded from $F$, consider those whose triple includes the edge $e$. Given a $p$-set $W \subset e$, at most $k-1$ other edges contain $W$. Hence there are fewer than $\left(\begin{array}{c}k-1 \\ 2\end{array}\right)$ events of the form $A(W,\{e, x, y\})$. Since there are $\left(\begin{array}{c}r \\ p\end{array}\right)$ choices for $W$, fewer than $\left(\begin{array}{c}k-1 \\ 2\end{array}\right)\left(\begin{array}{c}r \\ p\end{array}\right)$ events have $e$ in their set of three edges. The same holds for $f$ and $g$, so $A(U,\{e, f, g\})$ is mutually independent of a set of all but at most $3\left(\begin{array}{c}k-1 \\ 2\end{array}\right)\left(\begin{array}{c}r \\ p\end{array}\right)$ events.

By these computations and the given hypothesis,

$$
e \operatorname{Pr}[A(U,\{e, f, g\})] \cdot 3\left(\begin{array}{c}
k-1 \\
2
\end{array}\right)\left(\begin{array}{l}
r \\
p
\end{array}\right)<3 \frac{1}{\left(\begin{array}{l}
r \\
p
\end{array}\right)^{2}} \cdot 3\left(\begin{array}{c}
k-1 \\
2
\end{array}\right)\left(\begin{array}{l}
r \\
p
\end{array}\right)<1,
$$

where $e$ is the base of the natural logarithm. By the Lováz Local Lemma (see, e.g., [1]), the probability that no event of the form $A(U,\{e, f, g\})$ occurs is positive.

For an orientation $\vec{H}$ of an $r$-uniform hypergraph $H$, let $m(\vec{H})=\max \left|d_{S}(U)-d_{T}(U)\right|$, where the maximum is over choices of $p$-sets $U, S, T$ such that $U \subset V(H)$ and $S, T \subset[r]$. Let $f_{p}(H)$ denote the minimum of $m(\vec{H})$ over all orientations of $H$; this is the imbalance of an optimal orientation. Let $f(p, r, n)$ denote the maximum of $f_{p}(H)$ over all $r$-uniform hypergraphs with $n$ vertices. By Theorem $1, f(1, r, n)=f(r-1, r, n)=1$. By Theorem 2, $f(2,4,6)>1$, for example. We state an open problem.

Problem 7. Is $f(p, r, n)$ always bounded by a value that is independent of $n$ ?

\section{Acknowledgment}

We thank an anonymous referee for a correction to Corollary 4.

\section{References}

[1] N. Alon and J. H. Spencer, The Probabilistic Method, 2nd Edition, (Wiley, 2000).

[2] L. Lovász and M.D. Plummer, Matching theory, (AMS Chelsea Pub., 2009). 